\title{
ENTREVISTA A MARÍA VICTORIA PERALTA, PREMIO NACIONAL DE EDUCACIÓN DE CHILE. UNA VIDA DEDICADA A LA INVESTIGACIÓN DE LA PRIMERA INFANCIA
}

Interview with María Victoria Peralta, National Education Award of Chile. A video dedicated to the investigation of early childhood

Entrevista para Maria Victoria Peralta, Prêmio Nacional de Educação do Chile. Uma vida dedicada à investigação da primeira infância

\begin{abstract}
Aldo Ocampo González
Centro de Estudios Latinoamericanos de Educación Inclusiva (CELEI)1, Chile aldo.ocampo.gonzalez@gmail.com

Concepción López-Andrada

Centro de Estudios Latinoamericanos de Educación Inclusiva (CELEI), Chile clopezc@unex.es

Francisco Gárate Vergara

Centro de Estudios Latinoamericanos de Educación Inclusiva (CELEI), Chile fjgaratevergara@gmail.com
\end{abstract}

\begin{abstract}
Resumen
Los medios han destacado después de la obtención del Premio Nacional de Ciencias de la Educación de Chile en el año 2019 que María Victoria Peralta es la primera Educadora de Párvulos en lograr este galardón. En su trayectoria profesional, ha ejercido como Educadora de Párvulos y Profesora de aula en el Sistema Escolar Nacional chileno, cabe señalar del mismo modo su labor en establecimientos en condición de vulnerabilidad. En el ámbito de

\footnotetext{
1 Primer centro de investigación creado en Chile y en América Latina y el Caribe (ALAC), dedicado al estudio teórico y metodologico de la educación inclusiva, articula su trabajo desde una perspectiva inter, posty para-disciplinar. Institución internacional acreditada por el Consejo Latinoamericano de Ciencias Sociales (CLACSO) y por el International Consortium of Critical Theory Programs (ICCTP), EE.UU.
} 
la formación de nuevas generaciones de educadoras y educadores es reconocida por su contribución en la formación inicial docente. Quienes han sido sus estudiantes, destacan y valoran su metodología de enseñanza desde una perspectiva propositiva, crítica y proyectiva. Ha sido Coordinadora Nacional de Educación Preescolar en el Ministerio de Educación de Chile (2002-2006), donde uno de sus logros más notables fue la creación de un plan de estudios nacional para la educación infantil (niñas y niños de cero a seis años de edad). Asimismo ha sido asesora del Gobierno de Chile en temas de la primera infancia en varios países de América Latina. En esta entrevista en profundidad se revisa algunos de sus aporten en relación a la formación inicial docente y se abordan problemáticas relacionadas con la diversidad, inclusión y la construcción de la ciudadanía en Latinoamérica.

Palabras claves: educación; inclusión; diversidad; interculturalidad; ciudadanía.

\begin{abstract}
The media have highlighted after obtaining the Chilean National Prize for Educational Sciences in 2019 that María Victoria Peralta is the first Kindergarten Educator to achieve this award. In her professional career, she has worked as a Kindergarten Educator and Classroom Teacher in the Chilean National School System, as well as in vulnerable schools. In the area of training new generations of educators, she is recognized for her contribution to initial teacher training. Those who have been her students highlight and value her teaching methodology from a propositive, critical, and projective perspective. She has been the National Coordinator of Preschool Education in the Ministry of Education of Chile (2002-2006), where one of her most notable achievements was the creation of a national curriculum for preschool education (girls and boys from zero to six years of age). She has also been an advisor to the Chilean Government on early childhood issues in several Latin American countries. This in-depth interview reviews some of her contributions in relation to initial teacher training and addresses issues related to diversity, inclusion and the construction of citizenship in Latin America.
\end{abstract}

Key words: education; inclusion; diversity; interculturality; citizenship.

\title{
Resumo
}


Os meios de comunicação destacaram a obtenção do Prêmio Nacional de Ciências da Educação do Chile no ano de 2019 que Maria Victoria Peralta é a primeira Educadora de Párvulos no logaritmo deste galardão. Na bandeja profissional, ele funciona como Educador de Párvulos e Professor de Aula no Sistema Escolar Nacional do Chileno, cabe de forma semelhante ao modo de trabalho no estabelecimento de condições de vulnerabilidade. No ambiente da formação de novas gerações de educadores e educadores, é possível reconfigurar sua contribuição na formação inicial docente. Quienes foram seus estudiosos, destacando e valorizando a metodologia deense desde uma perspectiva propositiva, crítica e proativa. Foi Coordenadora Nacional de Educação Pré-escolar no Ministério de Educação do Chile (2002-2006), onde um dos seus logotipos é mais notável pela criação de um plano de estudos nacionais para a educação infantil (crianças e meninos de seis anos de idade) edad). $\mathrm{O}$ asimismo foi assistido pelo Gobierno do Chile em temas da primeira infancia em vários países da América Latina. Nesta entrevista, em profundidade, revise os algunos de seus autores, relacionados à formatação inicial docente e aborda problemas relacionados à diversidade, inclusão e construção da cidadania latino-americana.

Palavras-chaves: educação; inclusão; diversidad; interculturalidade; ciudadanía.

\section{Bionota de la entrevistada}

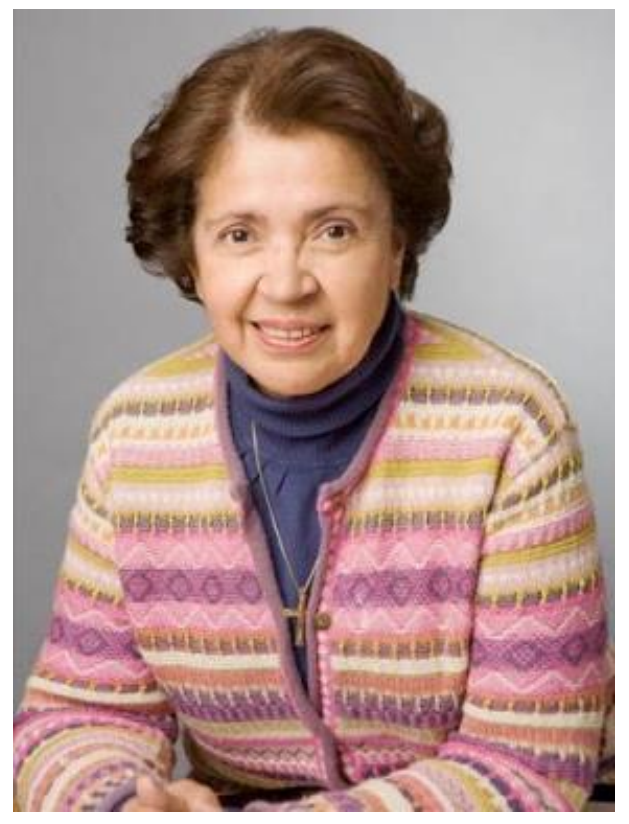

Académica con más de 40 años dedicados a la docencia e investigación en educación de la primera infancia. Educadora de párvulos y profesora de música de profesión, posee un Doctorado en Educación y es Magíster en Currículo Educacional y en Antropología Sociocultural. En su trayectoria se ha dedicado a evidenciar la importancia de la educación infantil en el desarrollo del potencial humano de niños y niñas, promoviendo el derecho a una educación oportuna y apropiada desde que se nace.

Una educación que permita a bebes, niñas y niños la posibilidad de desplegar sus capacidades de relación, 
indagación, creación e interpretación del mundo, donde el juego y el descubrimiento sean elementos fundamentales para la construcción de sus aprendizajes.

Se ha desempeñado como docente de cátedras de la especialidad en diversas universidades nacionales y de Latinoamérica. Fue Coordinadora y docente del primer programa de Magister en Educación Parvularia de Chile en la UMCE, publicando importantes libros en la materia, destacando la serie de ocho volúmenes de la "Historia de la educación infantil" y las publicaciones "Innovaciones Curriculares en Educación Infantil", "Una pedagogía de las oportunidades" por nombrar algunas.

Fue Directora de la Junta Nacional de Jardines Infantiles (1990-1998); Coordinadora del Componente Reforma Curricular de la Educación Parvularia, instancia donde se elaboraron las Bases Curriculares de la Educación Parvularia, y Coordinadora Nacional de Educación Parvularia del Ministerio de Educación.

Ha sido asesora por parte del Gobierno de Chile en casi todos los países latinoamericanos, y consultora de O.E.A., UNICEF, UNESCO, BID, PMA y O.E.I. Ha tenido el rol de Consultora Internacional en la elaboración de los Currículos Oficiales de Educación Inicial en Nicaragua, Ecuador, Paraguay, México, Panamá y Uruguay.

Francisco Gárate Vergara (F.G.V.): Buenos días, con mis compañeros del Centro de Estudios Latinoamericanos de Educación Inclusiva (CELEI), nos encontramos muy 
agradecidos por esta oportunidad de dialogar con usted. Para comenzar Dra. Peralta, ¿Desde su experiencia y estudios cuál es su concepto de diversidad e inclusión que le gustaría compartir con nosotras?

María Victoria Peralta Espinosa (M.V.P.E.): El concepto de diversidad: todos los seres humanos como dirían algunos filósofos, Mounier, entre otros. Somos personas únicas, un universo propio en un medio donde estamos con otros universos, que también tienen sus características, intereses, necesidades, sentidos, construcción de sentido es muy importante. Por lo tanto, esta diversidad humana que es tan inherente a lo que somos es lo que nos hemos reconocido y es el reconocimiento a la diversidad. Diversidad es eso, es esas características propias, peculiares, que lleva la humanidad en cada uno de nosotros, y que debemos tratar de fortalecer, conocer, optimizar, y hacer que descubramos otras diversidades en el camino, porque uno no es un ser con sus características determinado, sino por el transcurrir de la vida va descubriendo otras diversidades de su ser que también hay que incorporar. Eso sería para mí fundamentalmente diversidad, inclusión; obviamente favorecer, que existan, que podamos trabajar educativamente en conjunto todas estas diversidades, lo cual es un gran desafío aún.

Concepción López-Andrada (C.L.A.): Ya lo ha dicho variadamente, el rol que tiene que tomar la educadora y educador, el ejercicio en su quehacer profesional, pero ¿cómo cree que nuestra educación globalmente podría conseguir la inclusión?

M.V.P.E.: Globalmente, es lo que decía hace un rato, es formación de aptitudes, es formación de valores, pero a mí la diversidad y respecto a ella es un valor, y como todos los valores difíciles de formar, que se pierden a veces entre tantas, tantas otras expectativas que se quieren y que falta en esta gran definición. Porque si hay algo que nos falta en la sociedad chilena, es que no nos hemos hecho la pregunta ¿Qué tipo de sociedad queremos? Hemos pasado por ene reformas, contrarreformas y todo tipo de currículo, y la pregunta básica ¿Qué sociedad queremos? ¿Qué tipo de ser humano? ¿Qué niño o niña queremos favorecer? Y como respuesta de eso, un respeto entre otros aspectos a la diversidad, no ha sido contestada del todo y por eso es que no lo tenemos todos puesto como un norte importante de favorecer a través de nuestras prácticas en el hogar y en la escuela. 
Tener esa visión y esa sensibilidad para captar la diversidad en todos los planos, porque yo lo que percibo habitualmente, es que cuando se habla de inclusión y diversidad, se toman algunos aspectos, o sea, primero les ha costado mucho salir del enfoque un poco de necesidades educativas especiales. La mayoría de las personas, de los educadores, de las familias ¿cierto? Lo siguen mirando muchos por ese lado. Algo se ha metido el tema en algunos aspectos culturales, sobre todo ahora con el tema migración, ahí se ha visto porque es muy evidente la necesidad que tienen los niños, las familias, de integrarlas a la sociedad y a la escuela ha ido tomando esa dimensión, pero si nosotros tomamos el concepto y aquí me voy más bien a la interculturalidad en el sentido macro como el que tiene, plantea el concepto de UNESCO, en qué es la diversidad justamente en todos los planos desde opción sexual hasta religiosa, linguiística, cultural, social, características personales, capacidades distintas, bueno, eso pienso que todavía estamos bastante a años luz de eso. Por tanto, la educación debiera ser así, ese es el desafío.

Aldo Ocampo González (A.O.G.): En relación a las bases curriculares de nuestro país, ¿qué opinión tiene acerca del sentido de estas como eje de diversidad e inclusión?

M.V.P.E.: Mira, en las bases curriculares de la Educación Parvularia, el tema de la atención a los niños con sus necesidades, sus fortalezas, sus características, sus intereses, ha estado toda la vida; yo, tanto por mí formación como educadora de párvulos, como anteriormente. Si uno revisa los clásicos en educación, todos, no solamente en educación parvularia que eran los mismos con educación básica en el fondo, todos los grandes, todos hablaban de eso. Y en realidad sin tener todavía todo el conocimiento que se tiene hoy día, todos lo llevamos a la práctica en el conocimiento que se tenía en esos momentos.

Por lo tanto, en la revisión de los programas de Educación Parvularia, siempre estaba en esos términos, aunque, sin las herramientas suficientes quizás para poder llevarlo a cabo. Ahora, en las bases curriculares, tanto la primera versión la del 2001, está bastante manifiesto sin usarse en ese momento todavía el concepto inclusión, porque eso surge después justamente por la Ley de Inclusión, ahí es muy abierta toda la invitación a trabajar el tema de la interculturalidad. En esa época también se hablaba y, así está escrito, necesidades educativas especiales, y se incluyó un concepto potente de párvulo, por eso la 
inclusión se queda en el aspecto carencial, pero, no en descubrir también todos esos talentos, todas esas fortalezas que todos tenemos, y que sirven mucho también para equilibrar si es que hay algún aspecto que uno tuviera que, que un poco subrayar o mejorar, optimizar ¿no? En el desarrollo o en las relaciones humanas con otros.

En la versión actual aparecen los conceptos explícitos de interculturalidad, aparece el concepto de inclusión, se hace una breve referencia y se da, en cuanto a las orientaciones generales ese llamado a que bueno, todo educador puede desarrollar distintas formas de trabajo. Para asumir todas estas características y diversidades de los niños, también en una parte de los ámbitos y de los núcleos se hace una referencia a ello, quizás algunos aspectos de la segunda parte, la parte de organización curricular, donde se estructura mucho, donde se hace una organización por tramos se le llama a la nueva versión de las bases, lo cual a mi modo de ver fue una perdida respecto a las anteriores, las anteriores establecían dos ciclos; desde el nacimiento hasta los tres años aproximadamente y de tres a seis, pero, se señalaba abiertamente que el desarrollo del niño no, no era, no es a histórico y que depende de las experiencias hasta donde avanza el niño en un aspecto o en el otro. Pero este, creo que hubo un retroceso al marcar un poco esto al primer año, al segundo año, al tercer año, porque sabemos que el desarrollo no va así de esta forma, ni lineal, ni parejo para todos.

Pero como por otra parte y eso es lo que le digo yo a las alumnas, a las educadoras, se da libertad, en el sentido de poder hacer, seleccionar sus propios objetivos de aprendizaje, formularlos, escoger su forma de planificación, creo que por ese lado un educador que quiere atender esta inclusión en su diversidad con todas sus diferencias tan personales en cada uno, puede hacerlo, eso es un poco pienso, las posibilidades que dan estas bases.

F.G.V.: En referencia a la interculturalidad que usted tanto ha mencionado: ¿qué acciones se podrían seguir de su experiencia, estudios y de su conocimiento tanto en Chile como en la región latinoamericana?

M.V.P.E.: El incluir el tema de interculturalidad, más que temas, es el gran propósito en la educación porque en este mundo actual, de fronteras bajas, globalizado, la interculturalidad en todos los sentidos está permanente. Yo llevo en el tema junto con otros curriculistas, como mi querida maestra doña Viola Soto, tanto, treinta o más años trabajando en ello. ¿Y 
qué diría yo? He obtenido un poco de eso, primero que este es un tema de formación, de actitudes y de conocimientos en nosotros los profesores. Para eso pienso que hay que robustecer mucho más varios aspectos en el currículo de formación, y uno de los que yo he notado mas débiles es el tema cultural. Por lo menos a nivel de educación parvularia, toda la formación en neurociencias actualmente, en psicología es bastante fuerte y tiene un enfoque muy, en ese sentido, en esa área. Pero, el tema de obtener una óptica cultural que te permita respetar, reconocer, favorecer por ejemplo la cultura del otro es harto mas difícil, porque eso tiene que ver con la formación de actitudes y la formación de actitudes y sacarse el etnocentrismo que todos llevamos a veces demasiado marcado, pensando que nuestra cultura es la más valiosa y que queremos como mejor regalo, que lo desarrollen los demás, es algo muy serio. O sea, sacarse los prejuicios con las poblaciones, con los pueblos originarios, sacarse los prejuicios con los afrodescendiente en Chile. Entonces poder mirar al otro en una perspectiva de horizontalidad, de respeto mutuo, de intercambio de saberes y haceres para formar una verdadera interculturalidad, no es un tema fácil, hay que empezar con los niños, con los párvulos y seguirlo a lo largo de toda la escuela.

Nosotros hemos desarrollado en Chile, en América latina distintas experiencias, ya desde hace mucho tiempo que hemos estado tratando de favorecer lo que llamábamos currículo culturalmente pertinente, hemos hecho distintas experiencias por ejemplo, en JUNJI en el año 91'y 92 se planteó como criterio la pertinencia cultural, la de los currículos y, de ahí surgieron los primeros programas para poblaciones de los pueblos originarios, en los diez pueblos originarios en Chile, hecho con ellos, en co-construcción.

Lo mismo con las poblaciones de las comunidades de la Patagonia, se hizo todo un programa especial, jardines infantiles a distancia, se hizo el llamado al resto del país a ir dando pertinencia a los currículos, a la par de una formación en áreas de antropología sociocultural, yo diría que algo se ha avanzado, pero, en realidad hay que profundizar mucho más. Estos últimos años nosotros hemos estado trabajando bastante con ciertas comunidades, ciertas comunas, por ejemplo: en los últimos cinco, seis años hemos estado trabajando en la comuna de Castro, primero creando con todos los actores, los cultores de la cultura las comunidades educativas, haciéndonos la gran pregunta ¿Qué se necesita 
favorecer? ¿Que sería importante para que los párvulos de hoy tuvieran una mejor relación con las culturas locales? Que, como bien sabemos, están con problemas muy serios, perdiendo saberes, cosmovisiones, pero, aquí estoy hablando de las culturas locales, desde Huillichas hasta los distintos tipos de cultura chilota, porque no hay la cultura chilota, y frente a eso hemos llegado finalmente a la selección de un currículo abierto a partir de las bases curriculares de ciertos ejes culturales significativos, porque no se trata de folclorizar el currículo. Si no, se trata de señalar qué es importante en la formación de un niño hoy, aquí en relación a su cultura de pertenencia.

El niño chilote en el diagnostico hecho, tremendamente ¿cierto? impregnado e impresionado por las culturas que te entrega la televisión, los juegos, los videos, dejando mucho de su ser de su conocimiento de sus prácticas, bueno, y ahí las respuestas en las comunidades fue muy clara, valores, los valores de una cultura compartida, de lo que ha creado la cultura chilota, de sus saberes como decimos y de sus quehaceres. Ese trabajo que ha sido incluso ya, tiene hasta todo un informe, una evaluación, aquí esta bitácora de una travesía, llego hasta la confección de materiales didácticos pertinentes, hechos por los artesanos chilotes. Por ejemplo, este es un material que lo hicieron ellos a raíz de este currículo (muestra un texto de estudio), recogiendo los restos del bosque nativo, los restos porque ya casi no queda bosque nativo, recogieron estos restos de madera, madera noble, cipreses, lenga, los lijaron, los convirtieron, ellos lo llaman el lego, es como un mecano, la gracia que tiene este material por ejemplo, tiene olor a bosque nativo, hasta los olores han ido entrando en todo eso. Eso ha sido una experiencia y ahora estamos ya hace dos años trabajando en San Bernardo, donde ya tenemos un currículo culturalmente pertinente para San Bernardo, recogiendo la rica historia de San Bernardo que es tremendamente mas heterogénea que la de Chiloé, ahí participaron arqueólogos, historiadores, conocedores de la casa de la cultura, comunidades, personajes, los folcloristas, los poeta, que allá hay muchos, tratando de velar que eran los ejes principales de esas culturas que les interesaban y ahora ya estamos en la fase de formación continua a toda la comunidad educativa y empezando ya la implementación. Y estamos posiblemente por empezar en una región entera, estamos postulando a ello, pero eso ya va a ser un paso bastante mayor. Ya al 
desarrollar con toda una región un currículo para los niños y niñas de esa región, así es que esto se ha hecho aquí, pero, también en América latina se ha hecho a nivel nacional.

Hemos tenido la oportunidad de asesorar países en eso, y si ustedes observan y revisan el currículo nacional de Panamá y el de Uruguay, el que sale para la educación parvularia, desde el nacimiento hasta los seis años en el caso de Uruguay y del nacimiento hasta los cuatro años en el caso de Panamá, son currículos nacionales que parten por situarse ellos, como país, como cultura histórica y espacialmente, y a partir de ellos. Desarrollar realmente toda una línea de trabajo que se hizo con comunidades de maestros, con las culturas de los países, es decir que ¿se puede hacer una mayor interculturalidad en América latina? Sí, pero valorando y conociendo nuestras propias culturas.

\section{A.O.G.: Y enfocado a los niños y niñas ¿Cómo podemos empezar a construir una ciudadanía desde esta infancia y que sea critica, pero a la vez no sea excluyente?}

M.V.P.E.: Hoy día todos hablamos de educación parvularia en favorecer de una ciudadanía local y planetaria. Este currículo que mostraba aquí lo que pretenden es favorecer esa ciudadanía local, con los valores de esa ciudadanía local. Para favorecer autoestima, cultura de pertenencia, relación con las familias, con las comunidades, responsabilidad y sentido de comunidad, cuidado de ese medio ambiente desde todo punto de vista de su patrimonio, etc. Lo mismo también se extiende a la cultura planetaria, la Pacha mama que es la tierra y, que tenemos que cuidarla todos porque al paso que vamos, todos sabemos el problema que hay. Para ambas dimensiones los niños son maravillosos, les interesa tanto ser ciudadano local y ellos son los primeros si tú desarrollas actividades de tipo de cuidado del medio ambiente, de preservación, de reciclaje, de cuidar que no se rompa el arbolito, de no matar el insecto, están preocupadísimos, los niños de Chiloé, por ejemplo, están preocupadísimos de que las abejas están desapareciendo. Cuando fue la crítica y salió Chiloé a defender los mares que estaban llenos de cultivos que están absolutamente contaminando el agua y los demás seres marinos, los niños de los jardines salieron a marchar con sus familias y con sus educadoras, no en una línea de disputa agresiva, sino haciendo ver, ellos dibujaban sus mares con pedacitos azules y pedacitos rojos, que estaban con toxinas, que estaban la marea roja y que ellos no podían comer pescaditos sanos. Entonces los niños tienen una conciencia de 
ciudadanía, de responsabilidad, critica pro positiva diría yo, porque ellos no se quedan como nosotros en el puro discurso, sino que ellos hacen y eso es lo maravillosos, por lo tanto, si uno empieza desde tan pequeños con ellos puede favorecer mucho a una actitud de este tipo en nuestros niños, jóvenes y en nuestra ciudadanía en general.

C.L.A.: Y llevándolo a la coyuntura política presente en este proyecto de ley de Sala Cuna Universal, finalmente a través de un proceso de subvención incorporar a los niños ya de cuatro años, cinco años directamente en los colegios y sacarlos de los jardines y sala cunas. ¿Qué opinión tiene acerca de eso usted?

M.V.P.E.: Bueno, el proyecto en realidad es un proyecto para continuar aumentando la cobertura de atención, que hace ya un tiempo estaba un tanto parada, hay que decirlo, y en ese sentido es urgente, ya que, el país en uno o en otro gobierno de los recursos necesarios para eso. El tema que hay aquí detrás de este proyecto es un tema ideológico obviamente, es la visión desde donde yo financio la atención a los párvulos. Aquí el modelo tradicional que ha funcionado en el país, que es hacerlo desde instituciones del sector público, llámese JUNJI, INTEGRA, los jardines VTF y las escuelas, donde están los grupos de transición que es la mayor atención en Chile, ojo con eso, ese modelo se quiere un poco reemplazar por esta subvención, que distintos sostenedores asuman y hagan ellos la ampliación de esta cobertura. Para eso, una parte de ellos podrían ir a escuelas, por eso hay que decirlo porque también una fundación podría también pedir hogar de Cristo, hay muchas Fundaciones en educación parvularia y $\mathrm{ONG}^{\prime}$ s, esta subvención, y ellos están detrás de eso, pero quieren mejorar el proyecto.

El riesgo es que por ejemplo, que Chile no lo tiene, un sistema de supervisión, de asesoría técnica, esto puede llevar por la cercanía de muchos colegios que se van a interesar en extenderse a los más pequeños, puede tener el riego enorme de la sobre escolarización de la educación, desde los dos años, ojo, eso ya existe en educación parvularia actualmente, hasta en salas cunas y no como producto de este proyecto, y no es que yo esté a favor de este proyecto, pero quiero hacer ver que estos problemas de calidad en educación parvularia son los que no se conversan nunca, y siempre estamos en el tema de recursos financieros y, se nos van los gobiernos enteros en eso y no seguimos mirando lo que pasa al interior de 
jardines infantiles, salas cunas y las escuelas, nosotros nos hemos cansado de decir que las escuelas donde se hace la mayor atención a párvulos en Chile, que son los niños de cuatro a cinco y de cinco a seis, que casi se atiende, en el último caso un 95, 96 \%, la mayoría están sobre escolarizados y de una forma tremendamente inadecuada, no hay forma adecuada a eso porque al decir sobre escolarización quiere decir que en la escuelita tradicional, tipo bancaria según diría Paulo Freire y ¿por qué? porque entre otras cosas la subvención permite que hayan 45 niños trabajando con una educadora. Y yo eso lo he dicho en muchas columnas que he escrito, distintas participaciones y ningún gobierno tengo que decirlo, hace nada por eso, mientras esa educadora de párvulos tenga 45 niños no le va a quedar otra que tener niños sentados con papel y lápiz haciendo palotes, porque no se pueden ni mover en una sala, entonces está bien, hay que preocuparse de este proyecto que tiene muchas imperfecciones, y además que en la práctica y, en eso han hecho análisis la CUT y otros, como son tan poco los establecimientos que hasta el momento se han podido reconocer educativamente, porque aquí había muchos jardines que arreglar primero, va a llegar un grupo muy pequeño. Esto yo personalmente pienso que el Estado ha sido un mejor actor en esto a través de sus instituciones.

Pero el tema es más complejo, no es solo el proyecto, nosotros hemos visto la educación parvularia siempre desde un paradigma de la simplicidad, si yo muevo dos cositos lo arreglo. Esto hay que verlo en forma sistémica, hay un montón de factores entre ellos lo de financiamiento que afectan a favor y en contra, pero el gran tema es justamente la calidad, y ahí tenemos problemas ya hace mucho tiempo, y estamos haciendo muy poco por eso. Por lo tanto, creo que alguien tiene en un minuto que tomar todo esto y verlo con una visión macro y atender a todos los temas que están endeudados.

A.O.G.: Y en la formación inicial de educadores, educadoras, profesores y profesoras ¿Que desafíos vendrían para ellos en esta nueva sociedad del siglo XXI que se quiere construir?

M.V.P.E.: A mí me tiene un poco ahogada la formación de educadores dentro del sistema tan normado, tan pauteado que tenemos actualmente en Chile, y esto se junta un poco con qué concepto de universidad tenemos. Yo siempre le digo a mis alumnas, fíjate que la 
generación mía, yo me forme en una universidad que teníamos tres años de duración de la carrera, pero teníamos unos profesores maravillosos, carrera académica; profesores comprometidos que estaban en el medio generando leyes, apoyando a los niveles políticos, metidos en los problemas, y nosotros nos sentíamos muy orgullosos de todo eso. En formación de disciplina teníamos un tercio quizás de lo que reciben hoy día los estudiantes, pero, había una apuesta sobre valores, sobre el compromiso profesional, sobre lo que era ser un profesional tremendo.

Toda esta maraña de competencia que se ha armado hoy día, estas mil pautas que se aplican, que yo las he revisado varias veces, conozco carreras de educación parvularia muy de adentro porque generalmente me invitan y estoy con ellas, conozco a mis colegas y veo muchas veces que no hay una correspondencia entre las evaluaciones y lo que son muchas escuelas. Hay algo aquí, yo creo que hay un sistema que hay que revisar, me gustaría al estudiante en trabajos de verano, de invierno, en las poblaciones, en los campos, ahí es donde uno aprende, ahí es donde uno conoce la pobreza y sus muchos problemas, otras diversidades, ¿cómo puedo aprender todo metida entre cuatro paredes? Entonces, yo en mi época teníamos eso, de hacer mucho trabajo de campo de verdad y nos íbamos un mes entero a trabajos de verano, de invierno, de otoño, de todo, sin dejar de estudiar mucho, muchos seguíamos estudiando con mucha seriedad. Pero realmente en trabajo de campo, viendo la realidad de los niños de las familia y de las comunidades; entonces la siento muy de libro, la siento de toneladas de conocimientos que después el estudiante no sabe mucho cuando está en una comunidad determinada ponerlo en práctica, por lo tanto, yo tengo una mirada crítica hacia la formación en general de todos los profesionales en Chile, algo pasa, que hemos perdido el motor, el impulso, el compromiso social, la responsabilidad, el atender realmente las necesidades de nuestras familias y sus niños.

F.G.V.: ¿Y con respecto a la formación continua de los mismos educadores, educadoras, profesores y profesoras?

M.V.P.E.: En cierta medida, hay algo de eso, que en los mismos lugares donde están ellos deberían hacerse los proyectos para formación. Dados por ellos mismos como por las instituciones y por el sistema. En este momento a un profesor, yo lo veo con otros 
profesores, educadoras que estudian post grados, post títulos, quieren hacer mil cosas, pero no pueden porque está el sistema evaluativo, tanto los estándares, tantos desempeños que tienen que cumplir, el marco de buena enseñanza que le dice esto otro, ante unos mapas de progreso espantosos que tenían que aplicar, entonces realmente hay nuevamente este contra sentido, un profesor que quiere hacer muchas cosas y que tiene ideas pero no lo dejan ser el profesional que tiene que ser, y en eso hay que hacer también un cambio importante, yo creo que el sistema en algunas cosas nos está ahogando.

Es obvio que hay que tener ciertos criterios de calidad para asegurar que los niños no tengan peligro y unas serie de cosas, pero como se les ha dado todo hecho muchas veces, me acuerdo de un programa que había de escritura, lectura escritura y matemática en que decía hasta las frases que la educadora tenía que decir, y usted tomaba un postgrado y ese profesor está hablando de hacer innovación curricular, de hacer diagnósticos. Entonces tenemos serios problemas en toda la formación de profesores, inicial y continua. Ahora, que hay gente que supera eso y se las arregla finalmente, comunidades y directores que hacen el empeño y logran tener argumentos para que les den esa mayor libertad lo hay, pero antes eso lo teníamos mucho más, esa libertad.

C.L.A.: ¿Y con respecto a que desafíos se podrían tener o recomendaciones para lograr una educación para la justicia social?

M.V.P.E.: La educación para la justicia social, debería ser su gran propósito, justicia social desarrollando la humanidad maravillosa que tienen todos los niños, las niñas, todos nuestros educando, pero, para esa justicia social hay que estar, lo que decía en terreno, hay que ver las necesidades que tienen, las comunidades, como viven, lo que requieren, lo que piensan, lo que quieren, sus expectativas que a veces son distintas que las nuestras, tanto correctas si es que se puede hablar algo de correcto porque eso es desde un punto de vista, pero lo que para ellos es válido. A veces, las comunidades que hay, por ejemplo, que los niños efectivamente aprenden a leer y escribir a los dos años ¿por qué es válido?, no desde el punto de vista de lo que sabemos que aprende un niño, es válido porque dentro de su proyección piensa que el niño mientras más luego aprende a leer y escribir le va a ir mejor en la escuela, en la vida y eso es por su propia experiencia, que siente que va luchando 
contra un mundo que es difícil; entonces ahí es cuando entra un profesor, o una educadora a darle a conocer el que niño retiene que tener de estructuras previas para aquellos que no es importante, el mecanicismo sino la comprensión de un texto, de un mensaje. En la formación de ciertas habilidades, bueno, vamos dando justicia a la educación, si es que la calidad favorece justicia social, si es de calidad, pero esa es la dolorosa calidad que yo siento que todavía estamos a medio camino.

Bueno, yo siento que para todo esto que hemos hablado, hay un tema que quisiera profundizar un poquito más, que es el concepto de universidad en este país. También yo siento a la universidad prisionada, algo lo dije hace un rato, pero la universidad es el faro, eso me enseñaron mis maestros, una luz que se investiga, donde se reflexiona, donde hay propuestas, donde se actúa, donde se está presente. Yo siento que en la universidad lo hemos dejado bastante de lado, muchas son universidades de mercado que responden a lo que el sistema les pide. La universidad actualmente no dice lo que tienen que decir, calla muchas veces. Más que nada llamados de atención, porque ese es nuestro error, es decir, mire por acá no estamos bien, esto habría que trabajarlo de otra forma, aquí hay debilidades fuertes en esto.

Las políticas públicas yo no siento siempre que están construyéndose sobre el saber de la investigación y la experiencia. Chile es un país maravilloso en el sentido que hemos tenido una historia en la educación, educadores sensacionales, maestros, de primer nivel, pero los educadores, todas las últimas décadas ya no se les escucha, son otros los que toman las decisiones en el ámbito de la educación y poder saber que es educación con todas sus complejidades, son años y años de estudios en los que estamos todos tratando de aportar un poco más. Por lo tanto, esta universidad en general como país, que la siento a veces formadora de técnicos en el sentido de técnicos, con respuestas hechas, no me parece que es lo que Chile necesita, ni para la formación de profesores ni ninguna de las otras profesiones que existen. Creo que hay que replantearse, en América Latina hay una búsqueda y hay grupos y movimientos, yo trato de participar en algunos de ellos en que estamos en este remirar las universidades. Es bien difícil porque el sistema está muy pauteado en todo esto, pero, la universidad debe ser, lo que fue en Chile mucho tiempo, la universidad que va 
alumbrando los caminos del saber $\mathrm{y}$, del bien común con toda su experiencia su investigación y su trabajo comprometido.

C.L.A.: muchas gracias, profesora Peralta, por esta estimulante conversación.

M.V.P.E.: gracias a ustedes.

\section{Referencias Bibliográficas}

Peralta, M.V. (2008). Innovaciones curriculares en educación infantil. Avanzando a propuestas posmodernas. México: Edit. Trillas.

Peralta, M.V. (2005). Nacidos para ser y aprender. Buenos Aires: Edit. Infanto-juvenil.

Peralta, M. V. (2002). Una pedagogía de las oportunidades: nuevas ventanas para los párvulos latinoamericanos del siglo XXI. Santiago: Edit. Universitaria. 